\title{
TWO-FLUID CALCULATIONS WITH CONDUCTION OF X-RAY EMISSION FROM ACCRETING NONMAGNETIC DEGENERATE DWARFS
}

\author{
J. N. Imamura*, R. H. Durisen*, D. Q. Lamb ${ }^{+\dagger}$, \\ and G. J. Weast ${ }^{+\dagger}$ \\ *Department of Astronomy \\ Indiana University \\ tDepartment of Physics \\ Massachusetts Institute of Technology \\ tDepartment of Physics \\ University of Illinois
}

\section{INTRODUCTION}

Kylafis and Lamb (1979, hereafter $\mathrm{KL}$ ) have recently completed a comprehensive study of $x$-ray emission from accreting nonmagnetic degenerate dwarfs. Their calculations assumed a common temperature for the electrons and the ions, and neglected electron conduction. However, when Compton cooling of the X-ray emission region becomes important at high masses, electron conduction begins to play a role (Kylafis 1978).

Here we report the results of calculations which allow the temperatures of the electrons and the ions to differ and which include electron conduction. We confirm that such a treatment is required for accurate results whenever Compton cooling greatly dominates bremsstrahlung cooling in the emission region. With no nuclear burning, Compton cooling dominates in a small region of parameter space (Katz 1977, KL). We find that two-fluid calculations are needed only in the smaller region defined by $M \geq 1.2 M_{O}$ and $M \geq 10^{-2} M_{E}$, where $M_{E}$ is the accretion rate at which gravitational and radiation forces balance. Thus the approach of $\mathrm{KL}$, who considered this case, is justified. With nuclear burning, however, Compton cooling dominates throughout most of parameter space (Katz 1977, Weast et al. 1979). We find that two-fluid calculations are then needed when $\bar{M} \geq 0.4 \mathrm{M}_{0}$ and $\approx 10^{-4} \mathrm{M}_{\mathrm{E}}$.

our results show that the $\mathrm{X}$-ray spectrum is softer but the $\mathrm{X}$-ray luminosity is larger when a two-fluid treatment is used. However, the changes are generally $\leqslant 258$. Electron thermal conduction is less important than one might expect. We illustrate these points by comparing the results of one- and two-fluid calculations for a $1.4 \mathrm{M}_{0}$ star accreting at a rate of $0.3 \mathrm{M}_{\mathrm{E}}$ without nuclear burning.

\section{CALCULATIONS}

Our calculations assume (1) steady, spherically symmetric accretion, (2) no magnetic field, (3) complete ionization of the accreting matter, 
and (4) either no nuclear burning, or steady burning at the accretion rate. These assumptions are the same as those of $\mathrm{KL}$ and Weast et al. (1979). However, here we allow for differing electron and ion temperatures and include electron conduction.

With these assumptions, the following picture of $\mathrm{x}$-ray emission by degenerate dwarfs emerges. Matter freely falling from infinity forms a standoff shock above the stellar surface (Hoshi 1973, Aizu 1973). The shocked plasma has a temperature of $10^{8}-10^{9} \mathrm{~K}$ and emits $\mathrm{X}$-rays, due to thermal bremsstrahlung, as it cools and settles onto the stellar surface. Roughly half of the X-rays are emitted outward and produce the observed hard X-ray flux; the other half are emitted inward and intercept the stellar surface, where they are reflected or absorbed. The resulting blackbody flux from the stellar surface appears as UV and soft X-radiation. Without nuclear burning, the postshock plasma cools by thermal bremsstrahlung except for high-mass ( $M \geq 1$ Mo) stars where compton cooling by the blackbody flux dominates. With nuclear burning, the blackbody flux is much larger and compton cooling dominates for all masses.

In either case, when compton cooling is very large, the Compton cooling time scale may be less than the electron-ion energy exchange time scale. The electrons are then cooled so rapidly that they are unable to achieve a common temperature with the ions. The rapid Compton cooling also reduces the standoff distance of the shock. The electron temperature gradient increases and electron conduction becomes significant. Under these circumstances, accurate results require a twofluid treatment with conduction of the $\mathrm{x}$-ray emission region.

So far, we have implicitly assumed that the shock is produced by coulomb collisions. This assumption is not as critical as one might suppose. Provided the shock does not convert a significant amount of energy into waves or nonthermal particles, the structure of the emission region and the resulting $X$-ray spectrum are not sensitive to the jump conditions at the shock. We have demonstrated this by computing twofluid models without conduction in which we assumed that the ratio $\beta$ of the electron and ion temperatures just behind the shock equaled 1 and 1/1836. The spectra in the two cases were nearly identical. The spectral fluxes differed by only 38 at $200 \mathrm{keV}$ and by less than a factor of 4 even at $1 \mathrm{MeV}$.

our calculations are carried out as follows. We use the Chandrasekhar mass-radius relation for degenerate dwarfs, and assume a composition $\mathrm{X}=0.7, \mathrm{Y}=0.3$ for the accreting plasma. We determine the temperature and density structure of the emission region by imposing the two-fluid jump conditions (Shafranov 1957) at the ion shock, and integrating the two-fluid hydrodynamic equations inward subject to the boundary conditions $v=v_{f f}, T_{i}=T_{e}=0, J=0$ at $r \gg R$ and $v \rightarrow 0$, $T_{i}=T_{e}=T_{b b}, J=0$ at $r=R$, where $v_{f f}$ is the freefall velocity, $T_{i}$ and $\mathrm{T}_{e}$ are the ion and electron temperatures, $\mathrm{T}_{\mathrm{bb}}$ is the stellar blackbody temperature, and $\mathrm{J}$ is the conductive flux. Radiation pressure is taken into account. Bremsstrahlung, Compton cooling, and conduction are included in the emission region. Iterations are performed until a selfconsistent value for the compton cooling rate is obtained. We use a Monte Carlo technique to treat degradation of the $X$-ray spectrum and the albedo of the stellar surface (Kylafis 1978). 
Our results show that the choice of boundary condition $J=0$ at $r=R$ is supported by several physical arguments. This effectively rules out the possibility suggested by Fabian, Pringle and Rees (1976) and invoked by King and Lasota (1979) of solutions in which conduction transports most of the accretional energy into the star, quenching much of the hard $\mathrm{X}$-ray emission.

\section{RESULTS}

Figures 1-3 show the structure of the emission region for a $1.4 \mathrm{M}_{\odot}$ star accreting at a rate of $0.3 M_{E}$ without nuclear burning. 1 The accretion rate corresponds to an electron scattering optical depth $\tau_{\text {es }}=14$ from the emission region to infinity. This model shows the largest difference in electron and ion temperatures and the largest conductive flux that we find assuming no nuclear burning. As such it represents an extreme case, but it is typical of what we find if there is burning.

Figure 1 compares the temperature profile in the emission region of the one-fluid calculation with those of the two-fluid calculation. The standoff distance of the shock in the former case is $8.9 \times 10^{5} \mathrm{~cm}$ and in the latter $1.1 \times 10^{6} \mathrm{~cm}$. In the one-fluid calculation, the shock temperature $\mathrm{T}_{\mathrm{S}}=196 \mathrm{keV}\left(2.3 \times 10^{9} \mathrm{~K}\right)$ whereas the electron and ion temperatures just behind the ion shock in the two-fluid calculation are $T_{e}=97 \mathrm{keV}$ and $T_{i}=336 \mathrm{keV}$.

Figure 2 and 3 show the thermal bremsstrahlung and Compton cooling rates in the emission region of the two-fluid calculation. Figure 2 also shows the enthalpy and conductive fluxes $F_{H}$ and $J$, whereas Figure 3 also shows the divergences of these fluxes $\nabla \cdot F_{H}$ and $\nabla \cdot J$. Compton cooling clearly dominates throughout most of the emission region. The ratio of $F_{H}$ and $J$ is a measure of the relative importance of conduction, and shows that it is significant throughout the emission region but never dominates. The value of $F_{H}$ ahead of the electron precursor represents the kinetic energy of the freely falling plasma. Since $J=0$ ahead of the precursor and $J=F_{H}=0$ at the stellar surface, conduction and bulk motion only transport energy from one part of the emission region to another, whereas thermal bremsstrahlung and compton cooling represent real energy losses. Nevertheless, Figure 3 allows us to compare the local heating and cooling due to the former with the cooling due to the latter. Note that $\nabla \cdot F_{\mathrm{H}}$ and $\nabla \cdot J$ are zero in front of the precursor and at the stellar surface.

On the basis of the temperature profiles shown in Figure 1, the emission region can be conveniently divided into three parts: (A) a precursor or preshock region, (B) a two-temperature $\left(T_{e} \neq T_{i}\right)$ region, and $(C)$ a single-temperature $\left(T_{e} \simeq T_{i}\right)$ region. The electron temperature in region $A$ is nearly equal to its postshock value due to thermal conduction. However, the electron denisty here is relatively low and very little thermal bremsstrahlung and Compton cooling takes place. This region therefore has little effect on the postshock structure or on the $\mathrm{X}$-ray spectrum that is produced.

\footnotetext{
We inadvertantly omitted radiation pressure from this particular calculation. If included, it would reduce the one-fluid shock temperature $T$ and the two-fluid ion shock temperaure $T_{i}$ by 308 , thereby altering other quantitles by similar amounts. quantitative comparisons between the results for the one- and two-fluid calculations should still be val1d.
} 


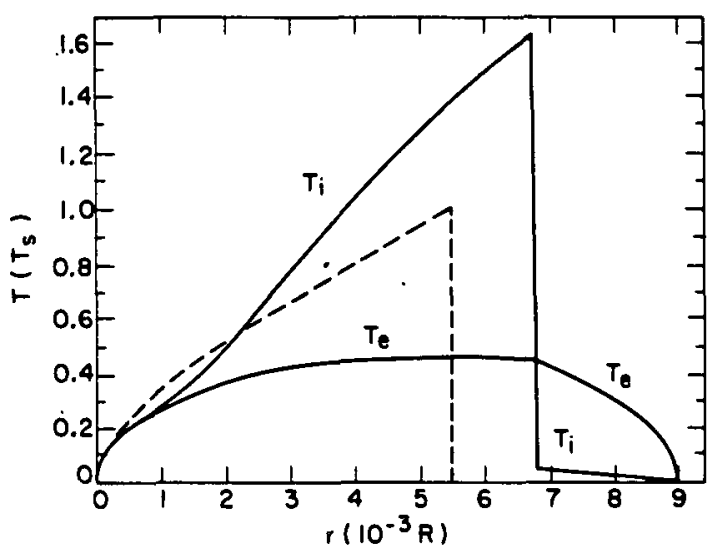

Fig. 1.- Comparison of temperature profiles in the $x$-ray emission reglon of a $1 M$ star accreting at a rate $0.3 \dot{\mu}$ without nuclear burning. The dashed curve shows the temperature profile in the one-fluid calculation, and the solid curves labeled $T$ and $T$. show the electron and lon temperature profiles in the two-fluid calculation. The temperatures are in units of thg one-fluid shock temperature $T=2.3 \times 10^{9} \mathrm{X}$ and the height $I$ above the stellar surface is in units of the stellar radius $\mathrm{R}=1.6 \times 10^{8}$ cm. The emission region can be convenientIy divided into three parts: (A) a preshock region, (B) a two-temperature $\left(T_{*} \neq\right.$ $T_{\text {, }}$ region, and (C) a one-temperature $\left(t_{e}=T_{1}\right)$ region.

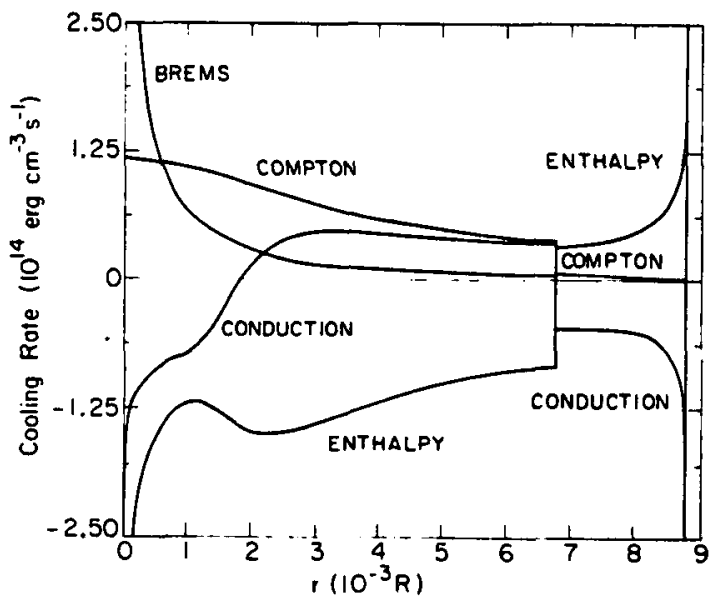

Fig. 3.- The thermal bremsstrahlung and Compton cooling rates, and the divergen$\operatorname{ces} \nabla \cdot F_{\text {and }}$ V.J of the enthalpy and conductive fluxes in the $\mathrm{X}$-ray emission region of the two-fluid calculation shown in Fig. 1. Positive values correspond to cooling, negative values to heating.

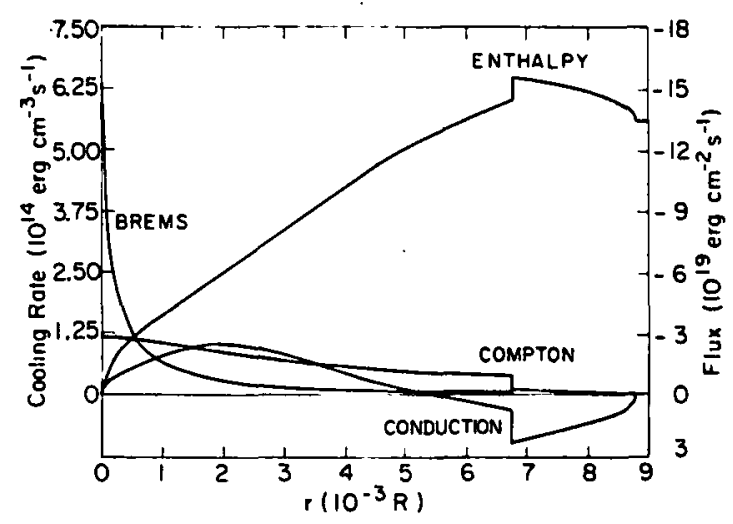

Fig. 2.- The thermal brensstrahlung and Compton cooling rates, and the enthalpy and conductive fluxes $F$ and $J$ in the $x$-ray emission region of the two-fluid calculation shown in Fig. 1. Positive - values of the rates correspond to cooling, negative values to heatingi positive fluxes are directed away fron the star, negative toward it.

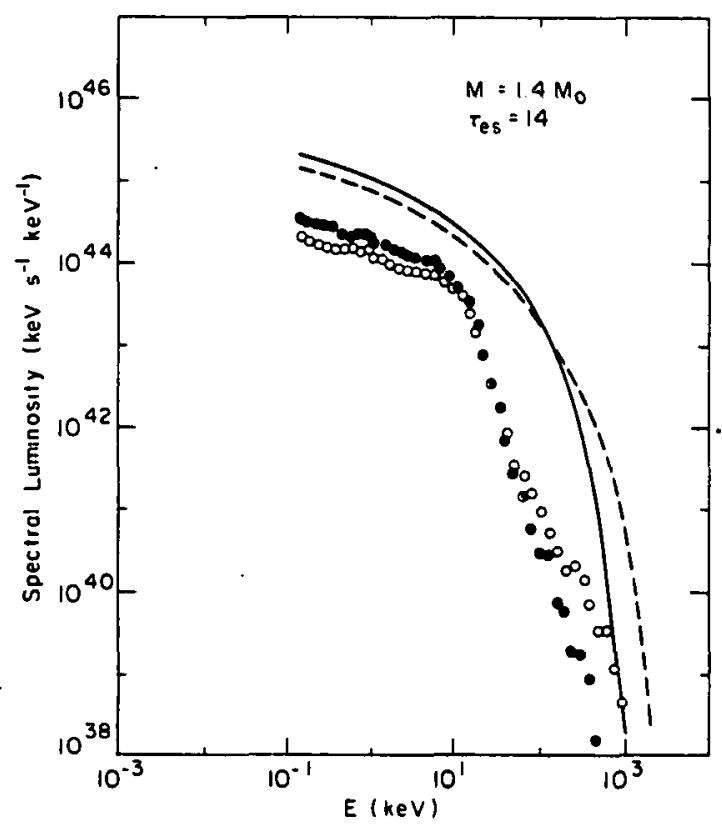

Fig. 4. - Comparison of the hard X-ray spectra produced by the one- and two-fluid calculations shown in Fig. 1. Key: spectrum produced in the emission region, o o o degraded spectrum observed at infinity in the one-fluid calculation, spectrum produced in the emission region, - - degraded spectrum observed at infinity in the two-fluid calculation. 
Although region B comprises most of the emission region, very few $X$-rays are produced in this region because bremsstrahlung emission is dominated by both Compton cooling and conduction (see Figure 3). The electron temperature here is determined by the balance between ion heating, and Compton and conductive cooling. These processes are independent of $T_{i}$, and hence $B$. The structure of the emission region and the resulting $x$-ray spectrum are therefore insensitive to the value of $B$, as we demonstrated earlier. The value of the electron temperature just behind the shock is $\simeq$ half that in the one-fluid calculation (see Figure 1). This has several consequences. The Compton cooling rate is smaller $\left(2.6 \times 10^{37} \mathrm{erg} \mathrm{s}^{-1}\right.$ versus $\left.3.0 \times 10^{37} \mathrm{erg} \mathrm{\textrm {s } ^ { - 1 }}\right)$ and the thickness of the emission region is therefore about 208 larger. The lower temperature and the larger emission region reduce the temperature gradient so that conduction is less important than one might expect. Lastly, fewer hard $\mathrm{X}$-rays are produced by the star because the maximum electron temperature in the emission region is smaller.

Thermal bremsstrahlung is larger than compton cooling in region $C$ and the bulk of the $X$-rays are therefore produced here. Because conduction transports energy from region $B$, where Compton cooling dominates, to region $C$, where thermal bremsstrahling dominates, $30 \%$ more $x$-rays are produced in the two-fluid calculation $\left(1.7 \times 10^{37}\right.$ erg $\mathrm{s}^{-1}$ compared to $1.3 \times 10^{37} \mathrm{erg} \mathrm{s}^{-1}$ ). Since conduction deposits energy in the coolest region near the stellar surface, the resulting $\mathrm{X}$-ray spectrum is softer.

Figure 4 compares the X-ray spectra produced in the one- and twofluid calculations. The spectrum produced in the emission region is softer in the two-fluid calculation: the spectral flux is 508 larger at $10 \mathrm{keV}$, and factors of 2 and 400 smaller at $200 \mathrm{keV}$ and $1 \mathrm{MeV}$. As this $X$-ray spectrum passes through the accreting plasma above the shock, it is degraded by Compton scattering. Figure 4 also compares the degraded $X$-ray spectra which are observed at infinity. The degraded spectra are little different, a bremsstrahlung fit giving an observed temperature $T_{o b s}=10$ and $8 \mathrm{keV}$ in the one- and two-fluid cases. Both degraded spectra show a power law tail, but the softer initial spectrum in the two-fluid calculation essentially eliminates the further high energy excess which was present in the degraded spectrum in the onefluid calculation. Since the example illustrated represents the most extreme case when there is no nuclear burning, and the typical case when there is burning, we conclude that the changes are generally $\$ 25 \%$. The dramatic spectral variations and the correlation between Tobs and $\mathrm{L}_{\mathrm{h}}$ reported by $\mathrm{KL}$ are little changed, and similar behavior should occur when there is nuclear burning (Weast et al. 1979).

In summary, we find that a two-fluid treatment including conduction is required for accurate results whenever compton cooling greatly dominates bremsstrahlung cooling in the emission region. The $x$-ray spectrum is softer but the $\mathrm{X}$-ray luminosity is larger when a two-fluid treatment is used. However, the changes are generally $\leqslant 25 \%$. Electron thermal conduction is less important than one might expect.

This research has been supported in part by the NSF under Grant PHY78-04404 and by NASA under contract NAS5-24441. DQL gratefu11y acknowledges support fron the John Simon Guggenheim Memorial Foundation. 


\section{REFERENCES}

Aizu, K. 1973, Prog. Theoret. Phys., 49, 1184.

Fabian, A. C., Pringle, J. E., and Rees, M. J. 1976, M.N.R.A.S., $\underline{175}, 43$.

Hoshi, R. 1973, Prog. Theoret. Phys., 49, 776.

Katz, J. I. 1977, Ap. J., 215, 265.

King, A. R., and Lasota, J. P. 1979, preprint.

Kylafis, N. D. 1978, Ph. D. Thesis, University of Illinois (unpublished).

Kylafis, N.D., and Lamb, D. Q. 1979, Ap. J. (Letters), 228, L105. Shafranov, V. D. 1957, J.E.T.P., 5, 1183.

Weast, G. J., Durisen, R. H., Imamurà, J. N., Kylafis, N. D., and Lamb, D. Q. 1979, this volume. 